\title{
Functional divergence of human genioglossus motor units with respiratory-related activity
}

\author{
S. Tsuiki, T. Ono, Y. Ishiwata, T. Kuroda
}

Functional divergence of human genioglossus motor units with respiratory-related activity. S. Tsuiki, T. Ono, Y. Ishiwata, T. Kuroda. (C)ERS Journals Ltd 2000.

ABSTRACT: The genioglossus muscle has at least two types of motor unit with respiratory-related activity. Inspiratory motor units show phasic activity during inspiration, whereas inspiratory/expiratory motor units show phasic inspiratory activity superimposed on tonic activity. The purpose of this study was to investigate the physiological roles of these different genioglossus motor units.

The unitary activities of $\mathbf{1 2}$ inspiratory and 12 inspiratory/expiratory motor units were recorded using fine-wire electrodes during quiet nasal breathing in eight normal adult males. The mean interspike interval and the SD of successive spikes were calculated for inspiratory and inspiratory/expiratory motor units, respectively. Scattergrams of the mean interspike interval versus SD were constructed for the two groups of motor units.

The effects of changes in head position on the firing activity and the patterns of distribution of the mean interspike interval versus SD were significantly different for inspiratory and inspiratory/expiratory motor units.

These results suggest that the inspiratory and inspiratory/expiratory motor units have different functional roles in respiration; inspiratory motor units may be phasically active to counteract intraluminal negative pressure during inspiration, whereas inspiratory/expiratory motor units may be tonically active to maintain tongue posture.

Eur Respir J 2000; 15: 906-910.
Maxillofacial Orthognathics, Maxillofacial Reconstruction, Division of Maxillofacial/ Neck Reconstruction, Graduate School, Tokyo Medical and Dental University, Tokyo, Japan

Correspondence: T. Ono, Maxillofacial Orthognathics, Maxillofacial Reconstruction, Division of Maxillofacial/Neck Reconstruction, Graduate School, Tokyo Medical and Dental University, 5-45, Yushima 1-chome, Bunkyo-ku, Fax: 81358030203

Keywords: Genioglossus muscle human

motor unit

respiration

tongue

Received: August 121999

Accepted after revision January 122000

This study was supported by Grants in Aid for Scientific Research $(07407060$ and 09470467) from the Japanese Ministry of Education, Science, Sports and Culture.
The upper airway (UA) muscles play an important role in respiration by counteracting the intraluminal negative pressure that is generated during inspiration, and thereby prevent the UA from narrowing and collapsing [1]. The pharynx, which comprises a large portion of the UA, lacks rigid bony support since it is surrounded by soft tissue. For instance, the anterior wall of the pharynx is partly determined by the tongue, and its posture is controlled by the electromyographic (EMG) activity of both the extrinsic and intrinsic tongue muscles. Numerous studies have focused on aberrant function of the extrinsic tongue muscles, especially the genioglossus muscle, since abnormal activity of this muscle contributes to snoring and obstructive sleep apnoea. Both in experimental animals and humans the genioglossus shows rhythmic activity in pace with respiration as well as tonic activity during tongue protrusion [2]. Some genioglossus fibres run perpendicular to the pharynx, and therefore activation of these fibres may result in both advancement of the base of the tongue and enlargement of the UA space. Previous physiological studies have shown that the muscle fibres of UA dilator muscles have faster contractile properties and less resistance to fatigue than the diaphragm $[3,4]$. In addition, the genioglossus contains type I, type IIa and type IIb fibres [4, 5]. However, it is not yet clear which type of motor unit is responsible for the respiratory-related activity of the genioglossus.
It has recently been shown that there are at least two types of motor unit with respiratory-related activity in the human genioglossus [6]: inspiratory motor units (IMUs), which show phasic firing during inspiration; and inspiratory/expiratory motor units (IEMUs), which fire during both inspiration and expiration, with a greater instantaneous firing frequency during inspiration. Their different patterns of firing activity indicate that these two types of motor unit play different physiological roles with regard to the respiratory/related control of tongue movement but it is unclear whether the IMUs and IEMUs are heterogeneous. It is possible that the IMUs and IEMUs are homogeneous, and their firing patterns are differentially controlled under unknown conditions. The purpose of this study was to investigate whether the IMUs and IEMUs are heterogeneous and to determine whether there is functional divergence between these two types of motor units with respiratory-related activity.

\section{Materials and methods}

\section{Subjects and protocol}

Eight healthy males with a mean \pm SD age of $27.1 \pm 2.0$ were studied. None had a history of respiratory disease. Infor-med consent was obtained from each subject before the study. The subject was seated in a chair and assumed an 
unrestrained upright head position, which was defined as the natural head position. After determining the subjects natural head position, they were asked to tilt their head dorsally and ventrally, defined hereafter as the head-up and head-down positions, respectively. Head position was measured with a custom-made device attached to the zygomatic arch [6]. The subject was instructed to remain awake with both eyes open and to breathe through the nose with the mouth closed. The upper and lower teeth were to be held slightly apart in the resting mandibular position. A urethane-coated fine stainless-steel wire (diameter $0.03 \mathrm{~mm}$; Unique Medical, Tokyo, Japan) was inserted into the genioglossus with a 27-gauge needle for monopolar recording of the single-unit activity of the motor units. A reference electrode was placed on the left ear lobe. Nasal airflow was recorded simultaneously using a thermistor (TR-762T; Nihon-Kohden, Tokyo, Japan). All EMG signals were amplified (MEG-6116; NihonKohden), band-pass filtered at $0.05-3 \mathrm{kHz}$ and sampled at $10 \mathrm{kHz}$. Recording of single motor unit activity from the genioglossus was identified by tonic burst activity during tongue protrusion. Subsequently, IMUs were identified by phasic firing activity primarily during inspiration, and IEMUs by tonic firing activity during both inspiration and expiration, with a greater instantaneous firing frequency (IFF) during inspiration. A window discriminator was used to segregate one motor unit from others and to calculate the IFF. It was confirmed that all spikes belonged to single motor units by monitoring the spontaneous firing activity of the motor unit on the oscilloscope with the fast sweep. The spontaneous firing activity in both the IMUs and the IEMUs was recorded for 20 respiratory cycles in the natural head position and in the $30^{\circ}$ head-up and $30^{\circ}$ head-down positions. Respiratory and EMG signals were transferred to a personal computer (Macintosh Performa 5270; Apple Computer, Cupertino, CA, USA) for data analysis. The IFF was calculated by dividing $1,000 \mathrm{~ms}$ by the interspike interval in milliseconds of two consecutive spike discharges with respiratory-related activity. Changes in the IFF were determined by subtracting the IFF obtained in the natural head position from those obtained in the $30^{\circ}$ headup and $30^{\circ}$ head-down positions. Changes in the IFF of both the IMUs and IEMUs in response to gradual changes in head position were also studied.

\section{Data analysis}

The significance of differences in IFF between respiratory phases was assessed using the Kruskal-Wallis test and Dunn procedure (Chart version 3.5.2/s, MacLab System $($ R, ADInstruments, Castle Hill, Australia) was used to evaluate the discharge characteristics of the motor units during spontaneous respiration. The mean interspike interval $(\tau)$ and the SD of succeeding spikes were calculated [79] during inspiration for the IMUs, and during both inspiration and expiration for the IEMUs. Histograms of $\tau$ and SD for both the IMUs and the IEMUs were constructed to confirm that their distributions were normal by calculating values for the skewness and kurtosis of each histogram (data not shown) [10]. Thereafter, the scattergram of the two groups of $\tau / \mathrm{sD}$ points was constructed. Differences between the two groups of $\tau /$ sD points were evaluated by means of discriminant analysis: since variance/covariance matrices for the two groups were unequal $\left(\chi^{2}=18.67\right.$, degrees of freedom $=3, p=0.00032$, Box's M test), Mahalanobis's generalized distance of each group and Wilks' $\lambda$ were measured [10]. Statistical significance was taken a p-value of $<0.05$.

\section{Results}

A total of 24 genioglossus motor units (12 IMUs and 12 IEMUs) recordings were made. Figure 1 shows a representative record of the firing activities of IMUs and IEMUs in the natural head position. Figure 1a shows the typical firing activity of one of the 12 IMUs, which started firing $24.3 \pm 149.8 \mathrm{~ms}$ (mean \pm SD, range $-297-293 \mathrm{~ms}$ ) before the onset of inspiratory airflow. This IMU showed firing activity primarily during inspiration with a mean IFF of $14.4 \pm$ 4.1 Hz. Figure $1 \mathrm{~b}$ shows the typical firing activity of one of the 12 IEMUs, whose IFF during inspiration (15.3 \pm 2.3 $\mathrm{Hz}$ ) was significantly greater than that during expiration $(10.8 \pm 2.5 \mathrm{~Hz})$.

Table 1 summarizes the changes in the IFF for both IMUs and IEMUs when the head position was changed from natural to either $30^{\circ}$ head-up or $30^{\circ}$ head-down. The increase in the IFF for IMUs was significantly greater during inspiration than during expiration immediately before and after inspiration when the head position was changed from natural to 30 head-up $(\mathrm{p}<0.05)$. In contrast, there was no significant difference in the IFF for IEMUs between any of the respiratory phases in the $30^{\circ}$ head-up position ( $p>0.05)$. These findings suggest that the firing activities of IMUs and IEMUs are modulated differently in association with $30^{\circ}$ head-up tilt. With regard to the change in the IFF for both IMUs and IEMUs when the head position was changed from natural to $30^{\circ}$ headdown, there were no significant differences in the IFF for both the IMUs and IEMUs between any of the respiratory phases in the $30^{\circ}$ head-down position ( $p>0.05$ ).

Since the $\tau /$ sD points for the IEMUs during inspiration were not significantly different from those during expiration (Wilks' $\lambda=0.952, F(2,77)=3.15, p>0.05$ ), the data for the IEMUs during inspiration and expiration were combined. The $\tau /$ sD points for both the IMUs and IEMUs were then plotted (fig. 2). The groups of $\tau /$ SD points for the IMUs and IEMUs were significantly different (Wilks' $\lambda$ $=0.627, \mathrm{~F}(2,149)=2.996, \mathrm{p}<0.05)$.

Figure 3 shows representative changes in the IFF of an IMU and an IEMU in response to gradual changes in head position. This IMU/IEMU pair was recorded simultaneously. The IMU showed a marked increase in IFF during inspiration when the head was dorsally tilted $15^{\circ}$ and $30^{\circ}$. In the head-up position, the firing duration of the IMU extended to the expiratory phase immediately before and after the inspiratory phase when the head was dorsally tilted $15^{\circ}$, which resulted in an increase in the IFF during expiration. The IFF during expiration further increased in the $30^{\circ}$ head-up position. Conversely, the IFF of the IMU markedly decreased during both inspiration and expiration when the head was tilted ventrally. The firing activity of the IMU almost ceased in the $30^{\circ}$ head-down position. The change in the IFF of the IMU in response to gradual changes in head position followed a sigmoid curve. Seven of the 12 IMUs examined showed this pattern of change 
a)<smiles>CCCCCCCCCCCCCCCCCCCCCCCCCCCC</smiles>

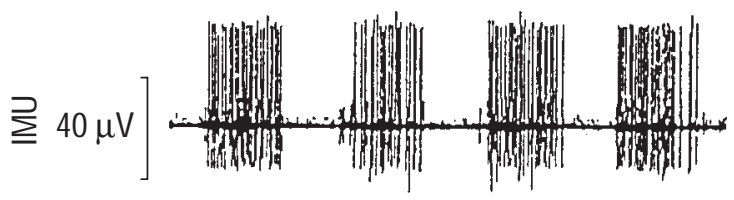

$\left.\begin{array}{ll}\stackrel{N}{1} & \\ \stackrel{4}{\underline{4}} & 0\end{array}\right]$

b)

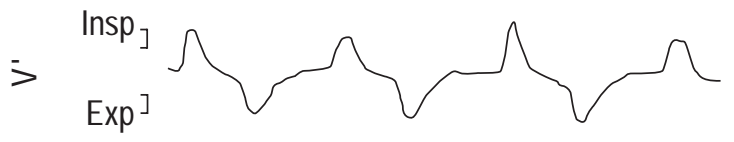<smiles>C#CC#CC12C3C4C(C#C)C5C3C1C5C42</smiles>

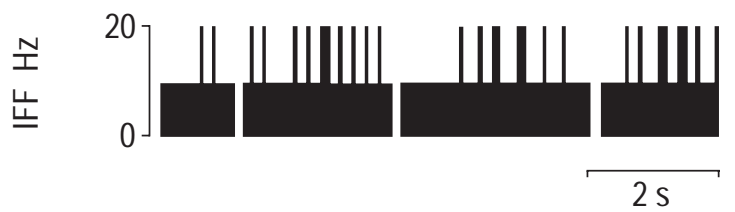

c)

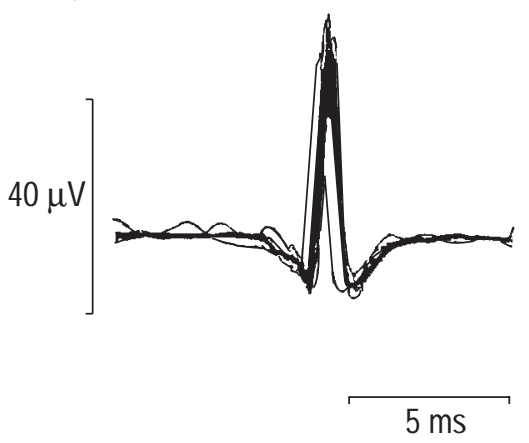

d)

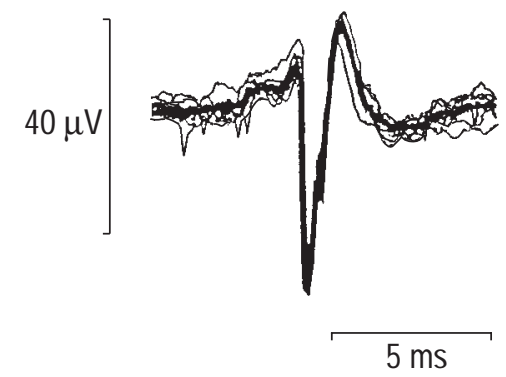

Fig. 1. - Typical firing activities of: a, b) an inspiratory motor unit (IMU); and c, d) an inspiratory/expiratory motor unit (IEMU). b) Superimposed spikes of the IMU shown in a; d) superimposed spikes of the IEMU shown in c (twenty sweeps are superimposed in b and d. $V^{\prime}$ : nasal airflow; Insp: inspiration; exp: expiration; IFF: instantaneous firing frequency (bin width $200 \mathrm{~ms}$ ).

in the IFF in response to gradual changes in head position. In contrast, the IFF of the IEMU gradually increased during both inspiration and expiration when the head was tilted dorsally. Conversely, the IFF of the IEMU gradually decreased during both inspiration and expiration when the head was tilted ventrally. In the $30^{\circ}$ head-down position, the IEMU still showed firing activity. The change in the IFF of the IEMU in response to gradual changes in head position was linear. All 12 of the IEMUs examined showed this pattern of change in the IFF in response to gradual changes in head position.

\section{Discussion}

The results of this study suggest that the IMUs and IEMUs have different functional roles in respiration.

Table 1. - Changes in instantaneous firing frequency (IFF) of inspiratory (IMU) and inspiratory/expiratory motor units (IEMUs) when the head was tilted from the natural to $30^{\circ}$ head-up and head-down positions

\begin{tabular}{|c|c|c|c|c|c|c|}
\hline & \multicolumn{3}{|c|}{ IMU } & \multicolumn{3}{|c|}{ IEMU } \\
\hline & Exp (pre) & Insp & Exp (post) & Exp (pre) & Insp & $\operatorname{Exp}$ (post) \\
\hline Natural Hz & $1.3 \pm 1.8$ & $15.6 \pm 3.8$ & $1.7 \pm 1.6$ & $16.4 \pm 1.3$ & $20.9 \pm 1.2$ & $17.0 \pm 2.0$ \\
\hline $30^{\circ}$ Head-up Hz & $16.7 \pm 3.2 *$ & $76.2 \pm 12.3 *$ & $19.5 \pm 1.4^{*}$ & $24.3 \pm 1.5^{*}$ & $28.2 \pm 2.2 *$ & $24.0 \pm 3.1 *$ \\
\hline$\Delta$ IFF head-up Hz & $15.4 \pm 2.8$ & $60.6 \pm 5.4^{+}$ & $17.8 \pm 1.6$ & $7.9 \pm 1.1$ & $7.3 \pm 1.4$ & $7.0 \pm 2.3$ \\
\hline $30^{\circ}$ Head-down $\mathrm{Hz}$ & $0.25 \pm 1.1 *$ & $1.0 \pm 0.4 *$ & $0.0 \pm 0.0^{*}$ & $9.5 \pm 2.1 *$ & $13.1 \pm 2.1 *$ & $10.2 \pm 2.4^{*}$ \\
\hline$\Delta$ IFF head-down $\mathrm{Hz}$ & $-1.05 \pm 1.4$ & $-14.6 \pm 3.3^{+}$ & $-1.7 \pm 1.6$ & $-6.9 \pm 1.6$ & $-9.6 \pm 1.7$ & $-6.8 \pm 2.2$ \\
\hline
\end{tabular}

Note that there was an increase in the IFF in the head-up position ( $\triangle \mathrm{IFF}$ head-up) of IMUs during inspiration, whereas there was no change in the IFF of IEMUs associated with $30^{\circ}$ head-up tilt. Exp (pre): expiration immediately before inspiration (Insp); Exp (post): expiration immediately after Insp; $\Delta \mathrm{IFF}$ head-down: change in IFF in the head-down position. *: $\mathrm{p}<0.05$ versus natural position; ${ }^{+}$: $\mathrm{p}<0.05$ versus expiratory phase. 


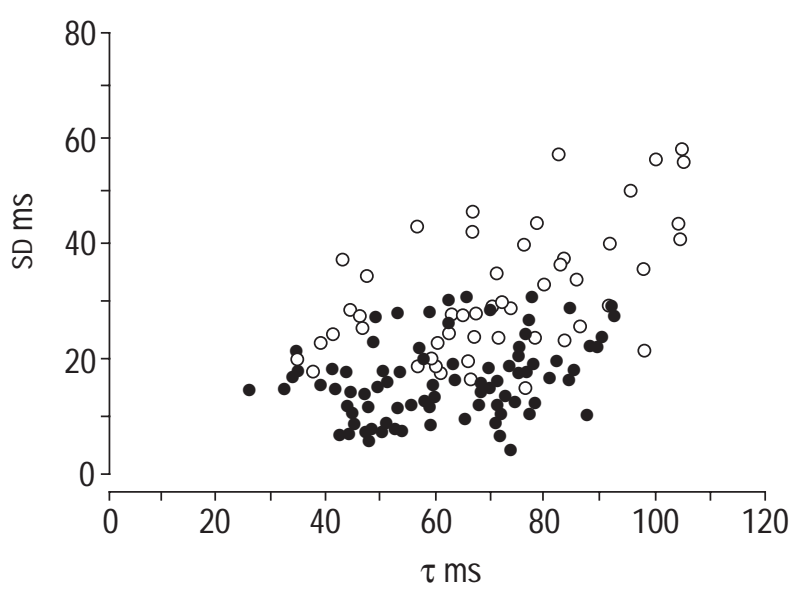

Fig. 2. - Mean interspike interval $\tau$ SD points for 12 inspiratory motor units (IMU; $\bigcirc$ ) and 12 inspiratory/expiratory motor units (IEMU; 0 ) $\tau$ and the SD were calculated during inspiration for the IMU, and during both inspiration and expiration for the IEMU.

Although the spatial distribution of fibre types in genioglossus is unknown, it is assumed that the various types of motor units have different roles since genioglossus is involved in different respiratory and nonrespiratory (e.g., mastication) functions. VAN LUNTEREN and coworkers [3, 4] reported that muscle fibres of pharyngeal dilator muscles such the genioglossus, geniohyoid, sternohyoid and sternothyroid showed fast contractile properties and resistance to fatigue. Hellstrand [11 ] found that the extrinsic muscles of the cat tongue contained 19-25\% type I fibres and $75-81 \%$ type II fibres. Although they only used a histochemical approach, they found that fatiguable, fatigue-intermediate and fatigue-resistant fibres were present in equal proportions in the extrinsic tongue muscles of the cat. BRACHER et al. [5] showed that there were $24 \%$ type I fibres and $47 \%$ type IIa fibres in the cat genioglossus whereas there were $11 \%$ type I fibres and $50 \%$

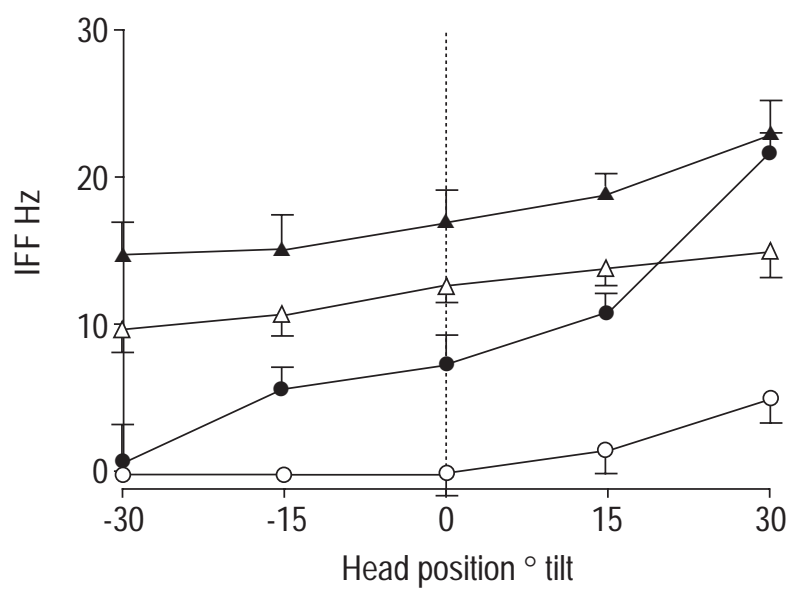

Fig. 3. - Representative changes in the instantaneous firing frequencies (IFFs) of an inspiratory motor unit (IMU) and an inspiratory/expiratory motor unit (IEMU) in response to gradual changes in head position. The firing activities of the IMU and IEMU were recorded simultaneously. Head position was gradually changed between the $30^{\circ}$ head-up and $30^{\circ}$ head-down positions. The IFF was calculated for both the IMU and IEMU in each head position: $\bigcirc$ : IMU during inspiration; $\bigcirc$ : IMU during expiration; $\boldsymbol{\Delta}$ : IEMU during inspiration; $\triangle$ : IEMU during expiration. type IIa fibres in the rat genioglossus muscle. LiN et al. [12] reported that the genioglossus was fatigue-resistant during tongue protrusion in the monkey. These reports suggest that extrinsic tongue muscle consists mainly of fatigue-resistant fibres. Unfortunately, there are few reports on fibre types in the human genioglossus. YAROM et al. [13] reported that tongue muscles (no differentiation among muscles was reported) were composed of $30-40 \%$ slow-twitch fibres in patients with Down syndrome. SERIES et al. [14] recently demonstrated that the genioglossus contains 33\% type I fibres and 67\% type II fibres in both snorers and patients with obstructive sleep apnoea. The two types of respiratory-related motor units in this study showed significantly different discharge patterns and were modulated differently in association with head-up tilt. Although there is no evidence regarding the precise relationship between types of muscle fibre and motor unit in the genioglossus, this difference suggests that the muscle is morphologically and functionally differentiated.

The hypoglossal nerve innervates muscles that protrude the tongue and reduce UA resistance and muscles that retract the tongue and increase UA resistance. Only a portion of hypoglossal motoneurons receive respiratory input from the central rhythm generator for respiration [15]. Since the firing activity of motor units indicates the excitation of the motoneurons per se, the classification of genioglossus motoneurons in terms of their respiratory-related activity may be feasible by examining the firing duration, timing and frequency of the motor units [6]. Lowe et al. [16] reported that the activity of motor units without respiratory-related discharge was modulated by electrical stimulation of the infraorbital, lingual, glossopharyngeal and superior laryngeal nerves. MILLER and BOWMAN [9] showed that the firing frequency of motor units increased during inspiration and swallowing using interspike interval analysis. However, there have been no studies on the functional role of respiratory-related motor units in humans. TOKIZANE et al. [7] examined the firing pattern of motor units in 46 muscles with respiratory-related units during tidal breathing. They demonstrated that the discharge pattern of respiratory-related units could be classified into two types, one that shows phasic activity during inspiration and another that shows tonic activity with greater firing frequency during inspiration [7]. The two types of motor units in the present study could be classified similarly; the IMU corresponds to the former type, and the IEMU to the latter.

It has been reported that human skeletal muscle contains both tonic and kinetic motor units [7]. ToKIZANE and SHIMAZU [8] speculated that tonic motor units are abundant in muscle that is involved in maintaining body posture (e.g. soleus muscle), whereas kinetic motor units are abundant in muscle that is involved in performing rapid movement (e.g. external ocular muscle). As shown in figure 2, the SD of the interval of spike discharge of IMUs was greater than that of IEMUs at a given inter-spike interval. The groups of $\tau$-SD points for IMUs and IEMUs were significantly different, suggesting that IMUs and IEMUs consist of heterogeneous subpopulations.

The firing activity of the IMU and the IEMU in the $30^{\circ}$ head-down position may provide insight into their divergent functional roles. In the 30 head-down position, the firing activity of IMUs almost ceased, whereas IEMUs remained active (fig. 3). Interestingly, IMUs became silent 
in the $30^{\circ}$ head-down position even during inspiration, whereas IEMUs maintained the same rate of firing activity during inspiration and expiration, regardless of head position. This suggests that IMUs and IEMUs are driven by different inputs. Gravitational pull in the head-down position would induce anterior displacement of the tongue, which would result in enlargement of the UA. Thus, it is assumed that IMUs are controlled mainly by commands from the central rhythm generator for respiration, whereas IEMUs are controlled mainly by peripheral feedback. This is consistent with previous studies in paralysed animals $[15,17,18]$, which showed that neither hypoglossal motoneurons nor their premotor neurons exhibited respiratory-related activity, which is analogous to the firing activity of IEMUs in the present study.

Although further studies are required on the input/output relationship in inspiratory and inspiratory/expiratory motor units, it appears that the respiratory-related activity of inspiratory motor units may phasically counteract the intraluminal negative pressure during inspiration. Conversely, the respiratory-related activity of inspiratory/expiratory motor units may tonically maintain tongue posture; otherwise the tongue would tend to collapse into the upper airway and jeopardize normal respiratory function, as occurs in patients with obstructive sleep apnoea [1].

\footnotetext{
Acknowledgements. The authors are grateful to T. Fukaya and S. Takahashi for their assistance
} in preparing the final version of the manuscript.

\section{References}

1. Isono AS, Remmers JE. Anatomy and physiology of upper airway obstruction. In: Kryger MH, Roth T, Dement WC, eds. Principles and Practice of Sleep Medicine, Philadelphia, W.B. Saunders, 1994; pp. 642-656.

2. Lowe AA. The neural regulation of tongue movements. Progr Neurobiol 1980; 15: 295-344.

3. Van Lunteren E, Salomone RJ, Manubay P, Spinski GS, Dick TE. Contractile and endurance properties of geniohyoid and diaphragm muscles. J Appl Physiol 1990; 69: 1992-1997.

4. Van Lunteren E, Manubay P. Contractile properties of feline genioglossus, sternohyoid, and sternothyroid muscles. J Appl Physiol 1992; 72: 1010-1015.

5. Bracher A, Coleman R, Schnall R, Oliven A. Histochemical properties of upper airway muscles: comparison of dilator and nondilator muscles. Eur Respir J 1997; 10: 990-993.

6. Tsuiki S, Ono T, Ishiwata Y, Kuroda T. Modulation of respiratory-related genioglossus motor unit activity associated with changes in head position in humans. Jpn J Oral Biol 1998; 40: 53-61.

7. Tokizane T, Kawamata K, Tokizane H. Electromyographic studies on the human respiratory muscles. Studies on the activity pattern of neuromuscular units. Jpn $J$ Physiol 1952; 2: 232-247.

8. Tokizane T, Shimazu H. Functional Differentiation of Human Skeletal Muscle, Tokyo, University of Tokyo Press, 1964.

9. Miller AJ, Bowman JP. Divergent synaptic influences affecting discharge patterning of genioglossal motor units. Brain Res 1974; 78: 179-191.

10. Hair JF, Anderson RE, Tatham RL, Black WC. Multiple discriminant analysis and logistic regression. In: Hair JF, Anderson RE, Tatham RL, Black WC, eds. Multivariate data analysis. New Jersey, Prentice-Hall, 1998; pp. 239325 .

11. Hellstrand E. Morphological and histochemical properties of tongue muscles in cat. Acta Physiol Scand 1980; 110: 187-198.

12. Lin L-D, Murray GM, Sessle BJ. The effect of bilateral cold block of the primate face primary somatosensory cortex on the performance of trained tongue-protrusion task and biting tasks. J Neurophysiol 1993; 70: 985-996.

13. Yarom R, Sagher U, Havivi Y, Peld IJ, Wexler MR. Myofibers in tongues of Down's syndrome. J Neurol Sci 1986; 73: 279-287.

14. Series F, Simoneau J-A, St. Pierre S, Marc I. Characteristics of the genioglossus and musculus uvulae in sleep apnea hypopnea syndrome and in snorers. Am J Respir Crit Care Med 1996; 153: 1870-1874.

15. Whithington-Wray DJ, Mifflin SW, Spyer KM. Intracellular analysis of respiratory-modulated hypoglossal motoneurons in the cat. Neuroscience 1988; 25: 10411051.

16. Lowe AA, Gurza S, Sessle BJ. Excitatory and inhibitory influences on tongue muscle activity in cat and monkey. Brain Res 1976; 113: 417-422.

17. Ono $\mathrm{T}$, Ishiwata $\mathrm{Y}$, Inaba $\mathrm{N}$, Kuroda $\mathrm{T}$, Nakamura $\mathrm{Y}$. Hypoglossal premotor neurons with rhythmical inspiratory-related activity in the cat: localization and projection to the phrenic nucleus. Exp Brain Res 1994; 98: 1-12.

18. Ono T, Ishiwata Y, Inaba N, Kuroda T, Nakamura Y. Modulation of the inspiratory-related activity of hypoglossal premotor neurons during ingestion and rejection in the decerebrate cat. J Neurophysiol 1998; 80: 48-58. 